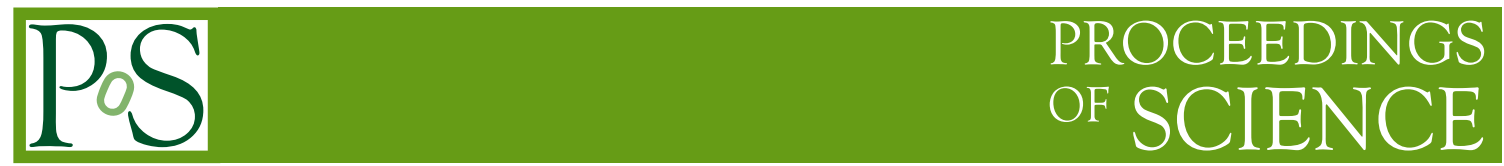

\title{
Helicity and infinite spin representations of the Poincaré group in 6D
}

\section{M.A. Podoinitsyn}

Bogoliubov Laboratory of Theoretical Physics, Joint Institute for Nuclear Research, 141980 Dubna, Moscow Region, Russia

E-mail: mpod@theor.jinr.ru

The massless irreducible representations of the Poincare group in the six-dimensional Minkowski space are investigated. We found convenient forms of the Casimir operators and analyzed their spectra. According to this analysis, we conclude that the helicity representation is defined by two (half-)integer numbers, while the infinite spin representation is determined by the real parameter $\mu^{2}$ and one (half-)integer number.

RDP online workshop "Recent Advances in Mathematical Physics" - Regio2020,

5-6 December 2020

online 


\section{Introduction}

In this letter we discuss the massless helicity and infinite spin irreducible representations of the Lie algebra of the Poincaré group in the 6D Minkowski space. At now such studies are motivated by an increased interest in field theories of higher dimensions. The attractiveness of such theories first of all arises due to their connection with the string theories, that may be interpreted as theories of infinite number of higher spin fields in higher dimensional space-time (see e.g. [1]).

The theory of unitary irreducible representations of the 4D Poincare group began in a series of works [2-4]. A large number of the aspects of unitary irreducible representations in higher dimensions were considered in the papers [5], [6], [8], [9], [10] and in lectures [11] (see also the recent paper [12]). The generic scheme of constructing the representations of the Poincare group in any dimension can be realized on the base of the method of induced representations (see e.g. [13], [14]).

Some details of the 6D Poincaré group representations are considered in papers [15],[16] however many moments, for example the infinite spin representations, were not addressed.

The letter is organized as follows. In Section 2 we discuss the Casimir operators and their properties in the 6D standard massless momentum reference frame. In Section 3 we analyze the massless finite spin irreducible representations and show that they are described by two (half-)integer numbers. Section 4 is devoted to infinite spin representations which are described by arbitrary real parameter and a single (half-)integer number. Section 5 is a summary of the results.

\section{Poincaré algebra and light-cone reference frame}

The Lie algebra iso $(1,5)$ of the Poincare group in the 6D space-time is given by the generators $P_{n}$ and $M_{m n}$ with the following commutation relations

$$
\begin{gathered}
{\left[P_{n}, P_{k}\right]=0, \quad\left[M_{m n}, P_{k}\right]=i\left(\eta_{m k} P_{n}-\eta_{n k} P_{m}\right),} \\
{\left[M_{m n}, M_{k l}\right]=i\left(\eta_{m k} M_{n l}+\eta_{n l} M_{m k}-\eta_{m l} M_{n k}-\eta_{n k} M_{m l}\right),}
\end{gathered}
$$

where the $D$-vector indices run the values $m, n=0,1, \ldots, 5$ and we use the mostly minus convention for the components $\eta_{m n}$ of the diagonal flat metric.

\subsection{Casimir operators of 6D Poincaré algebra}

We introduce the third rank tensor $W_{m n k}$ and the vector $\Upsilon_{m}$ as the elements of the universal enveloping algebra of $\mathfrak{i s o}(1,5)[5,15]$

$$
\begin{aligned}
W_{m n k} & =\varepsilon_{m n k l p r} P^{l} M^{p r}, \\
\Upsilon_{m} & =\varepsilon_{m n k l p r} P^{n} M^{k l} M^{p r},
\end{aligned}
$$


where $\varepsilon_{m n k l p r}$ is a totally antisymmetric tensor and $\varepsilon_{012345}=1$. Using commutation relations (1) and (2) one can check that the operators

$$
\begin{aligned}
C_{2} & :=P^{m} P_{m}, \\
C_{4} & :=\frac{1}{24} W^{m n k} W_{m n k}, \\
C_{6} & :=\frac{1}{64} \Upsilon^{m} \Upsilon_{m}
\end{aligned}
$$

are the Casimir operators of the algebra iso(1,5). By virtue of the expressions (3), (4) we obtain explicit form of the Casimir operators (5), (6), (7):

$$
\begin{aligned}
C_{2}= & P^{m} P_{m}, \\
C_{4}= & \Pi^{m} \Pi_{m}-\frac{1}{2} M^{m n} M_{m n} C_{2}, \\
C_{6}= & -\Pi^{k} M_{k m} \Pi_{l} M^{l m}+\frac{1}{2}\left(M^{m n} M_{m n}-8\right) C_{4} \\
& +\frac{1}{8}\left[M^{k l} M_{k l}\left(M^{m n} M_{m n}-8\right)+2 M^{m n} M_{n k} M^{k l} M_{l m}\right] C_{2},
\end{aligned}
$$

where we introduce a new $6 \mathrm{D}$ vector $\Pi$ with components

$$
\Pi_{m}:=P^{k} M_{k m} .
$$

Further we consider only the massless unitary representations of the algebra iso( 1,5$)$, i.e. we fix the quadratic Casimir operator (8) as follows:

$$
C_{2} \equiv P^{2}=P^{m} P_{m}=0 .
$$

\subsection{Casimir operators in the standart massless momentum reference frame}

Let us move to the light-cone basis where any $6 D$ vector $X^{m}=\left(X^{0}, X^{a}, X^{5}\right)$ has the light-cone coordinates $X^{m}=\left(X^{+}, X^{-}, X^{a}\right)$ which are determined by the formulas

$$
X^{ \pm}=\frac{1}{\sqrt{2}}\left(X^{0} \pm X^{5}\right), \quad X_{ \pm}=\frac{1}{\sqrt{2}}\left(X_{0} \pm X_{5}\right) \quad \Rightarrow \quad X^{ \pm}=X_{\mp} .
$$

We now restrict our considerations to the standard massless momentum reference frame. So we will consider only those states $|k\rangle$ on which the momentum operator acts as follows

$$
P^{m}|k\rangle=k^{m}|k\rangle
$$

where

$$
k^{m}=(\sqrt{2} k, 0,0,0,0,0), \quad k \in \mathbb{R} .
$$

The Casimir operators (9), (10) in the light-cone reference frame (14) take the form

$$
\begin{aligned}
& \hat{C}_{4}=-\hat{\Pi}_{a} \hat{\Pi}_{a}, \\
& \hat{C}_{6}=\hat{\Pi}_{b} M_{b a} \hat{\Pi}_{c} M_{c a}-\frac{1}{2} M_{b c} M_{b c} \hat{\Pi}_{a} \hat{\Pi}_{a},
\end{aligned}
$$


where we introduce operators

$$
\hat{\Pi}_{a}:=\sqrt{2} k M_{+a} .
$$

Taking into account (2) we can see that the operators $\hat{\Pi}_{a}$ and $M_{a b}$ from RHS of the formulae (16) and (17) form the Lie algebra of the $I S O(4)$ group

$$
\begin{aligned}
{\left[M_{a b}, M_{c d}\right] } & =i\left(\delta_{b c} M_{a d}-\delta_{b d} M_{a c}+\delta_{a c} M_{d b}-\delta_{a d} M_{c b}\right), \\
{\left[\hat{\Pi}_{a}, \hat{\Pi}_{b}\right] } & =0, \quad\left[\hat{\Pi}_{a}, M_{b c}\right]=i\left(\delta_{a b} \hat{\Pi}_{c}-\delta_{a c} \hat{\Pi}_{b}\right) .
\end{aligned}
$$

The operators $\hat{C}_{4}$ and $\hat{C}_{6}$ are the Casimir operators of the iso(4) algebra. Thus, in the massless case (12) the unitary irreducible representations of the 6D Poincare group are defined by the eigenvalues of the iso(4) Casimir operators (16) and (17). It can be verified that the $I S O$ (4) group is the small Wigner group (see [19]) or, in another terminology, a subgroup of stability of a massless momentum. Therefore, according to the Wigner scheme, massless irreducible representations of the $i s \mathfrak{s o}(1,5)$ can be induced from the irreducible representations of iso(4). There are two different cases of these irreducible representations of the iso(4) defined by the value of Casimir operator (16). So, in next sections we consider following unitary massless representations:

1. Helicity (finite spin) representations.

For these representations the four-vector $\hat{\Pi}_{a}$ has a zero norm:

$$
\hat{\Pi}_{a} \hat{\Pi}_{a}=0 .
$$

2. Infinite (continuous) spin representations.

Here the four-vector $\hat{\Pi}_{a}$ has a nonzero norm:

$$
\hat{\Pi}_{a} \hat{\Pi}_{a}=\mu^{2} \neq 0 .
$$

\section{Helicity representations}

From the condition (21), which determines this case, we immediately obtain the equations for the components of the vector $\hat{\Pi}_{a}$

$$
\hat{\Pi}_{a}=0 \quad \text { at all } \quad a=1,2,3,4 .
$$

Therefore, the Casimir operators (16) and (17) vanish for the case of the helicity representations. Consequently, such representations of $I S O(4)$ are finite dimensional, because noncompact $I S O(4)$ is reduced to compact group $S O(4)$. According to the Wigner scheme each such representation defines some $6 D$ massless representation.

\section{1 $6 D$ helicity operators}

In this section we will show that the Casimir operators of the stability subgroup $S O(4)$ define the $6 D$ helicity operators. Consider the vector $\Upsilon_{m}$ defined in (4). Using (7) if the constraint $C_{6}=0$ holds, we get $\Upsilon_{m} \Upsilon^{m}=0$. In the standart massless momentum reference frame the light-cone components of the $6 D$ vector $\Upsilon$ are

$$
\Upsilon^{+}=\Lambda_{1} P^{+}, \quad \Upsilon^{-}=\Upsilon_{a}=0,
$$


where we define

$$
\Lambda_{1}:=\epsilon_{a b c d} M_{a b} M_{c d} .
$$

It can be verified that this operator is the Casimir operator of the $\mathfrak{s o}(4)$ algebra.

From the conditions (24) we can conclude that the vectors $\Upsilon$ and $P$ are collinear, which means that the equality (in fact, this equality is valid in any reference frame)

$$
\Upsilon_{m}=\Lambda_{1} P_{m}
$$

This connection has already been noted in the works [15, 17]. Note that the operator (25) can be represented as follows

$$
\Lambda_{1}:=\frac{\Upsilon_{0}}{P_{0}} .
$$

Therefore, the operator $\Lambda_{1}$, defined in (27), is a $6 \mathrm{D}$ analog of the $4 \mathrm{D}$ helicity operator.

It is known that irreducible $\mathfrak{s o}(4)$ representations are characterized by two quadratic Casimir operators. The second helicity operator arises if we use the construction proposed in [17]. Namely, one can construct another vector with components ${ }^{1}$

$$
S_{m}:=3 M^{n k} P_{[m} M_{n k]} .
$$

In the standart massless momentum reference frame, the light-cone components of the $6 \mathrm{D}$ vector (28) are equal to (c.f. (24))

$$
S^{+}=\Lambda_{2} P^{+}, \quad S^{-}=S_{a}=0
$$

where we define

$$
\Lambda_{2}:=M_{a b} M_{a b} .
$$

This operator is the second Casimir operator of $\mathfrak{s o}(4)$. In a general frame, then the relations (29) take the form

$$
S_{m}=\Lambda_{2} P_{m},
$$

where the operator $\Lambda_{2}$ defines the second helicity operator, i.e. $\Lambda_{2}$ can also be represented in the form

$$
\Lambda_{2}:=\frac{S_{0}}{P_{0}} .
$$

Therefore, massless helicity representations are characterized by the pair $\left(\lambda_{1}, \lambda_{2}\right)$, where real numbers $\lambda_{1}, \lambda_{2}$ define the eigenvalue of the Casimir operators $\Lambda_{1}$ and $\Lambda_{2}$. Using some additional construction (see [7]), we can calculate the spectra of helicity operators $\Lambda_{1}$ and $\Lambda_{2}$.

$$
\begin{aligned}
& \lambda_{1}=8 j_{+}\left(j_{+}+1\right)-8 j_{-}\left(j_{-}+1\right), \\
& \lambda_{2}=4 j_{+}\left(j_{+}+1\right)+4 j_{-}\left(j_{-}+1\right),
\end{aligned}
$$

where $j_{ \pm}$are (half-)integer numbers.

\footnotetext{
${ }^{1}$ The definition of antisymmetrization has standart form, i.e.

$$
A_{\left[n_{1}\right.} B_{n_{2}} C_{\left.n_{3}\right]}=\frac{1}{3 !}\left(A_{n_{1}} B_{n_{2}} C_{n_{3}}+A_{n_{2}} B_{n_{3}} C_{n_{1}}+A_{n_{3}} B_{n_{1}} C_{n_{2}}-A_{n_{2}} B_{n_{1}} C_{n_{3}}-A_{n_{1}} B_{n_{3}} C_{n_{2}}-A_{n_{3}} B_{n_{2}} C_{n_{1}}\right) .
$$
}




\subsection{Two examples of the helicity representations}

Now we will use the obtained formulas (25) and (30) for determining the helicities for two massless finite spin fields. According the Wigner scheme the irreducible massless representations of the $6 D$ Poincaré group are induced by the irreducible $S O(4)$ representations.

Therefore, in this examples, we first consider a some irreducible $S O(4)$ representation and determine the eigenvalues of the helicity operators. Next, we consider the corresponding 6D fields. Fixing the gauge and using the $6 \mathrm{D}$ equations of motion in the standard massless momentum reference frame, we will show that $6 \mathrm{D}$ fields has the same of the independent components those $S O(4)$ fields which were considered earlier. That is in these example we will demonstrate the Wigner's strategy (scheme), which is very transparently presented in the form of points in the lectures [11].

Below, in this section we will use the standart notations for the $\mathfrak{s o}(4)$ generators in defining representation

$$
\left(\mathcal{M}_{a b}\right)_{e g}=i\left(\delta_{a e} \delta_{b g}-\delta_{a g} \delta_{b e}\right)
$$

\subsubsection{Vector field}

Consider the $S O(4)$ vector field $A_{a}$. Then, the helicity operators take the form

$$
\begin{aligned}
& \left(\Lambda_{1}\right)_{e g}=\epsilon_{a b c d}\left(M_{a b} M_{c d}\right)_{e g}=0, \\
& \left(\Lambda_{2}\right)_{e g}=\left(M_{a b} M_{a b}\right)_{e g}=6 \delta_{e g} .
\end{aligned}
$$

Acting on the $S O(4)$ vector field $A_{a}$, the operators (36) give the following values of helicities:

$$
\lambda_{1}=0, \quad \lambda_{2}=6 ; \quad j_{+}=j_{-}=\frac{1}{2} .
$$

Let us show that this Euclidean vector field $A_{a}$ describes physical components of the $6 D$ vector gauge field $A_{m}$. The massless gauge field $A_{m}$ is described by the 6D Maxwell equations

$$
P^{m} F_{m n}=0
$$

where $F_{m n}=i\left(P_{m} A_{n}-P_{n} A_{m}\right)$ is the standart definition of the field strength, and determined up to gauge transformations

$$
\delta A_{m}=i P_{m} \varphi .
$$

As an example, let us take the light-cone gauge (see e.g. [18]), i.e. $A^{+}=0$. Then, in the standard massless momentum reference frame, the light-cone equations of motion (38) are reduced to $A^{-}=0$. Therefore an independent field is given by the transverse part $A_{a}$ of the $6 D$ gauge field $A_{m}$.

\subsubsection{Second rank symmetric tensor field}

Let us consider the $S O(4)$ second rank tensors. Now the $\mathfrak{s o}(4)$ generators take the form

$$
\left(M_{a b}\right)_{e_{1} e_{2}, g_{1} g_{2}}=\left(\mathcal{M}_{a b}\right)_{e_{1} g_{1}} \delta_{e_{2} g_{2}}+\delta_{e_{1} g_{1}}\left(\mathcal{M}_{a b}\right)_{e_{2} g_{2}}
$$

and the helicity operators are

$$
\begin{aligned}
& \left(\Lambda_{1}\right)_{e_{1} e_{2}, g_{1} g_{2}}=8 \epsilon_{e_{1} e_{2} g_{1} g_{2}}, \\
& \left(\Lambda_{2}\right)_{e_{1} e_{2}, g_{1} g_{2}}=12 \delta_{e_{1} g_{1}} \delta_{e_{2} g_{2}}+4\left(\delta_{e_{1} g_{2}} \delta_{e_{2} g_{1}}-\delta_{e_{1} e_{2}} \delta_{g_{1} g_{2}}\right) .
\end{aligned}
$$


We consider the $S O(4)$ second rank tensor $\hat{h}_{a b}$, which is symmetric $\hat{h}_{a b}=\hat{h}_{b a}$ and traceless $\hat{h}_{a a}=0$. Acting by helicity operators (41) on this field we get the values

$$
\lambda_{1}=0, \quad \lambda_{2}=16 ; \quad j_{+}=j_{-}=1 .
$$

Note, that similarly to the vector case, we can conclude that field $\hat{h}_{a b}$ describes the physical components of the 6D linearized gravitational field.

\section{Infinite spin representations}

The case of the infinite spin representations is characterized by the condition (22). Then the representations of the $I S O(4)$ group are infinite dimensional.

In this case the Casimir operator (16) has a nonvanishing eigenvalue

$$
C_{4}=\hat{C}_{4}=-\mu^{2}, \quad \mu \neq 0 .
$$

So we can take the basis with a nonzero only fourth component:

$$
\hat{\Pi}_{1}=\hat{\Pi}_{2}=\hat{\Pi}_{3}=0, \quad \hat{\Pi}_{4}=\mu .
$$

In the case of the irreducible representations of infinite (continuous) spin, the Casimir operator (10) takes the value (for details see [7])

$$
C_{6}=\hat{C}_{6}=-\mu^{2} s(s+1),
$$

where $s$ is a (half-)integer number. As a result, the massless infinite spin representations are characterized by the pair $(\mu, s)$, where the real parameter $\mu$ defines the eigenvalue of the Casimir operator (43) and the (half-)integer number $s$ defines the eigenvalue of the Casimir operator (45).

\section{Summary}

In this letter we have investigated the massless irreducible representations of the Poincare group in the 6D Minkowski space. This irreducible representations are described by three Casimir operators. The properties of these operators have been analized in the standard massless momentum reference frame. Based on this analysis, one can see that the unitary representations of $\operatorname{ISO}(1,5)$ group are induced from representations of $S O(4)$ and $I S O(4)$ groups and, correspondingly, are divided into helicity (finite spin) and infinite (continuous) spin representations. It has been showed that the helicity representation is described by two (half-)integer numbers. The infinite spin representation is described by one real parameter and one (half-)integer number. For the case of helicity representations, we have presented two simple examples, in which the values of both helicity operators are explicitly calculated. All results obtained in this work are in good agreement with the statements of $[5,11,19]$.

\section{Acknowledgments}

The author is grateful to I.L. Buchbinder, S.A. Fedoruk and A.P. Isaev for formuation of the problem and useful discussions. This work was partialy supported by the Russian Foundation for Basic Research, project No. 19-01-00726A. 


\section{References}

[1] M.B. Green, J.H. Schwarz, E. Witten, Superstring theory, Cambridge Univ. Press, 1987.

[2] E.P. Wigner, On unitary representations of the inhomogeneous Lorentz group, Annals Math. 40 (1939) 149.

[3] E.P. Wigner, Relativistische Wellengleichungen, Z. Physik 124 (1947) 665.

[4] V. Bargmann, E.P. Wigner, Group theoretical discussion of relativistic wave equations, Proc. Nat. Acad. Sci. US 34 (1948) 211.

[5] L. Brink, A.M. Khan, P.Ramond, X.-Z.Xiong, Continuous spin representations of the Poincare and superPoincare groups, J. Math. Phys. 43 (2002) 6279, arXiv: hep-th/0205145.

[6] A.M. Khan, P. Ramond, Continuous spin representations from group contraction, J. Math. Phys. 46 (2005) 053515, arXiv: hep-th/0410107.

[7] I. L. Buchbinder, S. A. Fedoruk, A. P. Isaev, and M. A. Podoinitsyn, Massless finite and infinite spin representations of Poincaré group in six dimensions, Physics Letters B 813 (2021) 136064, arXiv:hep-th/2011.14725.

[8] X. Bekaert, N. Boulanger, On geometric equations and duality for free higher spins, Phys. Lett. B561 (2003) 183, arXiv: hep-th/0301243.

[9] X. Bekaert, N. Boulanger, Tensor gauge fields in arbitrary representations of $G L(D, R)$, Commun. Math. Phys. 271 (2007) 723, arXiv: hep-th/0606198.

[10] I. Bandos, X. Bekaert, J.A. de Azcarraga, D. Sorokin, M. Tsulaia, Dynamics of higher spin fields and tensorial space, JHEP 0505 (2005) 031, hep-th/0501113.

[11] X. Bekaert, N. Boulanger, The unitary representations of the Poincaré group in any spacetime dimension, Lectures presented at 2nd Modave Summer School in Theoretical Physics, 6-12 Aug 2006, Modave, Belgium, arXiv: hep-th/0611263.

[12] S. Weinberg, Massless Particles in Higher Dimensions, Phys. Rev. D102 (2020) 095022, arXiv:2010.05823 [hep-th].

[13] A.O. Barut, R. Raczka, Theory of Group Representations and Applications, Polish Scientific Publishing, 1977.

[14] A.P. Isaev, V.A. Rubakov, Theory Of Groups And Symmetries (I): Finite Groups, Lie Groups, And Lie Algebras. World Scientific, 2019.

[15] L. Mezincescu, A.J. Routh, P.K. Townsend, Supertwistors and massive particles, Annals Phys. 346 (2014) 66, arXiv: 1312 .2768 [hep-th].

[16] A.S. Arvanitakis, L. Mezincescu, P.K. Townsend, Pauli-Lubanski, supertwistors, and the super-spinning particle, JHEP 1706 (2017) 151, arXiv: 1601.05294 [hep-th]. 
[17] S.M.Kuzenko, A.E.Pindur, Massless particles in five and higher dimensions, arXiv:2010.07124 [hep-th].

[18] W. Siegel, Fields, arXiv:hep-th/9912205.

[19] X. Bekaert, J. Mourad, The continuous spin limit of higher spin field equations, JHEP 0601 (2006) 115, arXiv:hep-th/0509092. 